\title{
Effective and robust approach for fluorescence molecular tomography based on CoSaMP and $S P_{3}$ model
}

\author{
Xiaowei He*,*, Hongbo Guo*, Jingjing $\mathrm{Yu}^{\dagger}, \mathrm{Xu}$ Zhang* and Yuqing $\mathrm{Hou}^{*}$ \\ *Northwest University \\ School of Information Sciences and Technology \\ Xi'an, P. R. China 710069 \\ ${ }^{\dagger}$ Shaanxi Normal University \\ School of Physics and Information Technology \\ Xi'an, P. R. China 710062 \\ thexw@nwu.edu.cn
}

Received 6 November 2015

Accepted 17 December 2015

Published 11 February 2016

\begin{abstract}
Fluorescence molecular tomography (FMT) allows the detection and quantification of various biological processes in small animals in vivo, which expands the horizons of pre-clinical research and drug development. Efficient three-dimensional (3D) reconstruction algorithm is the key to accurate localization and quantification of fluorescent target in FMT. In this paper, 3D reconstruction of FMT is regarded as a sparse signal recovery problem and the compressive sampling matching pursuit (CoSaMP) algorithm is adopted to obtain greedy recovery of fluorescent signals. Moreover, to reduce the modeling error, the simplified spherical harmonics approximation to the radiative transfer equation (RTE), more specifically $S P_{3}$, is utilized to describe light propagation in biological tissues. The performance of the proposed reconstruction method is thoroughly evaluated by simulations on a 3D digital mouse model by comparing it with three representative greedy methods including orthogonal matching pursuit (OMP), stagewise OMP (StOMP), and regularized OMP (ROMP). The CoSaMP combined with $\mathrm{SP}_{3}$ shows an improvement in reconstruction accuracy and exhibits distinct advantages over the comparative algorithms in multiple targets resolving. Stability analysis suggests that CoSaMP is robust to noise and performs stably with reduction of measurements. The feasibility and reconstruction accuracy of the proposed method are further validated by phantom experimental data.
\end{abstract}

Keywords: Tomography; image reconstruction techniques; medical and biological imaging; inverse problems.

\$Corresponding author.

This is an Open Access article published by World Scientific Publishing Company. It is distributed under the terms of the Creative Commons Attribution 4.0 (CC-BY) License. Further distribution of this work is permitted, provided the original work is properly cited. 


\section{Introduction}

Fluorescence molecular tomography (FMT) is a sensitive and powerful whole body optical imaging approach. With specific fluorescence probe, FMT allows noninvasive detection and survey of molecular and cellular processes by accurately reconstructing three-dimensional (3D) distribution of the fluorescent targets. Thanks to extensive fluorescent probe research and improved data acquisition, FMT receives more and more attention in biomedical research community and expands the horizons of pre-clinical research and drug development. ${ }^{1-6}$

As an optical imaging modality, FMT has to cope with the fact that biological tissue is a highly scattering and absorbing medium. An accurate forward model of light transport is essential to reconstruct 3D distribution of the inside targets from the outside photon density detected on the surface. The radiative transfer equation (RTE) has been successfully used as a standard model for describing light propagation in biological tissues. ${ }^{7}$ Until now, providing solutions to RTE remains a challenging task for anisotropic biological tissue with spatially nonuniform scattering and absorption properties. Diffusion approximation (DA) to the RTE is one of the most widely used forward models in cases with low absorption coefficients or large geometries. Although DA is computationally efficient, it is less accurate in small animal imaging where the sourcedetector separation is small. Therefore, it is necessary to adopt some high-order approximation-based reconstruction methods to improve FMT reconstruction. When small tissue geometries and high light absorption are encountered, simplified spherical harmonics $\left(S P_{N}\right)$ method can accurately model light propagation and overcome the limitations of DA. ${ }^{8,9}$

The reconstruction of FMT is a severe ill-posed inverse problem and small perturbation of the charge-coupled-device (CCD) measurement will result in significant changes in reconstructed images. ${ }^{10}$ In order to deal with the ill-posedness of FMT and improve the reconstruction accuracy, regularization is essential in reconstruction procedure to generate meaningful and stable numerical solutions. ${ }^{11,12}$ By utilizing the fact that the fluorescence targets in many FMT scenarios are sparse in the sense that they typically occupy a small fraction of the overall imaging domain, researchers have proposed various compressed sensing (CS) inspired reconstruction algorithms for FMT.

Following the framework of CS, two major approaches to recover the unknown sparse targets are $\ell_{p}(0<p \leq 1)$ minimization and greedy methods. For example, Baritaux first propose a general $\ell_{p}(1 \leq p<2)$ regularization $\operatorname{method}^{11}$ and validate the adequacy of $\ell_{1}$ regularization for FMT reconstruction. And then several algorithms with $\ell_{1}$ penalty function have been reported, including the iterated shrinkage based algorithm, ${ }^{13}$ adaptive support driven reweighted $\ell_{1}$ regularization algorithm, ${ }^{14}$ incomplete variables truncated conjugate gradient method (IVTCG), ${ }^{15}$ and iteratively reweighted regularization method. ${ }^{16}$ More recently, nonconvex $\ell_{p}(0<p<1)$ regularizations are also investigated in FMT. By converting $\ell_{p}(0<p<1)$ minimization into a series of $\ell_{1}$ problems, nonconvex $\ell_{p}(0<p<1)$ methods exhibit good performance in accurately recovering sparse targets and sparsity enhancement. ${ }^{17,18}$ Although the CS theory demonstrates that the $\ell_{1}$ approach provides exact reconstruction in the noiseless case, ${ }^{19}$ greedy methods have more advantages in computational efficiency, especially for large-scale applications. Consequently, several greedy reconstructions based on orthogonal matching pursuit (OMP), ${ }^{20}$ stagewise OMP (StOMP) ${ }^{21}$ and adaptive matching pursuit $(\mathrm{AMP})^{22}$ have been applied to FMT. However, these greedy algorithms do not provide global convergence guarantees. ${ }^{23}$ In Ref. 23, Needell and Tropp developed a stable greedy algorithm, compressive sampling matching pursuit (CoSaMP), which bridged the gap between $\ell_{1}$ minimization and greedy methods. ${ }^{24,25}$ So far, however, there has been little investigation about CoSaMP in FMT.

The major concern of this paper is to present effective and robust reconstruction method for FMT. To improve the efficiency and accuracy of FMT reconstruction, $S P_{3}$ model is used to describe light propagation and the iterative CoSaMP algorithm is used to recover the unknown fluorescence signal. The proposed reconstruction method is implemented in the framework of finite element method (FEM). Simulations on a 3D digital mouse model and experimental evaluation on a physical phantom were conducted to investigate the performance of the CoSaMP algorithm combined with $S P_{3}$ model in FMT. 


\section{Methods}

\section{1. $\mathrm{SP}_{3}$ model}

For steady-state FMT with point excitation sources, the following coupled $S P_{3}$ equations have been extensively used to depict the photon propagation $^{9,26}$ :

$$
\left\{\begin{array}{l}
-\nabla \cdot \frac{1}{\mu_{a 1}} \nabla \varphi_{1}+\mu_{a} \varphi_{1}-\frac{2}{3} \mu_{a} \varphi_{2}=S \\
-\nabla \cdot \frac{1}{7 \mu_{a 3}} \nabla \varphi_{2}+\left(\frac{4}{9} \mu_{a}+\frac{5}{9} \mu_{a 2}\right) \varphi_{2} \\
-\frac{2}{3} \mu_{a} \varphi_{1}=-\frac{2}{3} S
\end{array}\right.
$$

where $\varphi_{1,2}$ is the composite of the Legendre moments of radiance, $\mu_{a i}=\mu_{a}+\left(1-g^{i}\right) \mu_{s}$, with $\mu_{a}$ the absorption coefficient, $g$ the anisotropy parameter, and $\mu_{s}$ the scattering coefficient. $S$ is the source term. To solve the Eq. (1), the Robin-type boundary conditions are widely used on the boundary ${ }^{27}$ :

$$
\left\{\begin{aligned}
\left(\frac{1}{2}+\right. & \left.A_{1}\right) \varphi_{1}+\frac{1+B_{1}}{3 \mu_{a 1}}\left(\mathbf{n} \nabla \varphi_{1}\right) \\
& =\left(\frac{1}{8}+C_{1}\right) \varphi_{2}+\frac{D_{1}}{\mu_{a 3}}\left(\mathbf{n} \nabla \varphi_{2}\right) \\
\left(\frac{7}{24}+\right. & \left.A_{2}\right) \varphi_{2}+\frac{1+B_{2}}{7 \mu_{a 3}}\left(\mathbf{n} \nabla \varphi_{2}\right) \\
& =\left(\frac{1}{8}+C_{2}\right) \varphi_{1}+\frac{D_{2}}{\mu_{a 1}}\left(\mathbf{n} \nabla \varphi_{1}\right)
\end{aligned}\right.
$$

The coefficients $A_{i}, B_{i}, C_{i}$ and $D_{i}$ are the constant coefficients related to the refractive indices of the biological tissue. ${ }^{28}$

In the FEM framework, the $S P_{3}$ model can be linearized and the following matrix-form equation can be obtained:

$$
\left[\begin{array}{ll}
M_{1 \varphi_{1}} & M_{1 \varphi_{2}} \\
M_{2 \varphi_{1}} & M_{2 \varphi_{2}}
\end{array}\right]\left[\begin{array}{l}
\varphi_{1} \\
\varphi_{2}
\end{array}\right]=\left[\begin{array}{c}
S \\
-\frac{2}{3} S
\end{array}\right] .
$$

For the excitation process, the excitation light source is modeled as isotropic-point sources located at one-mean-free path of photon transport beneath the surface. For the emission process $S=\Phi X, \Phi=$ $\varphi_{1 e}-\frac{2 \varphi_{2 e}}{3}$ is the total fluence, where $\varphi_{1 e}$ and $\varphi_{2 e}$ can be directly obtained by solving Eq. (3) in the excitation process, and $X$ is the unknown nodal value of the fluorescent yield to be reconstructed. So in the emission process, the following equation can be derived:

$$
\left[\begin{array}{ll}
M_{1 \varphi_{1}} & M_{1 \varphi_{2}} \\
M_{2 \varphi_{1}} & M_{2 \varphi_{2}}
\end{array}\right]\left[\begin{array}{l}
\varphi_{1} \\
\varphi_{2}
\end{array}\right]=\left[\begin{array}{ll}
\varphi_{1 e} & \\
& \varphi_{2 e}
\end{array}\right]\left[\begin{array}{c}
X \\
-\frac{2}{3} X
\end{array}\right]
$$

The measured quantity is the exiting partial current $J^{+}$which can be calculated by ${ }^{27}$

$$
\begin{aligned}
J^{+}= & \left(\frac{1}{4}+J_{0}\right) \varphi_{1}-\left(\frac{0.5+J_{1}}{3 \mu_{a 1}}\right) \mathbf{n} \varphi_{1}, \\
& -\left(\frac{1}{16}+\frac{2 J_{0}-J_{2}}{3}\right) \varphi_{2}-\left(\frac{J_{3}}{7 \mu_{a 3}}\right) \mathbf{n} \nabla \varphi_{2} .
\end{aligned}
$$

According to Eq. (2), $\mathbf{n} \cdot \nabla \varphi_{i}$ can be represented as a linear combination of $\varphi_{i}$. Consequently, the linear relationship between the measurement $J_{m}^{+}$and the unknown fluorescent yield distribution $X$ is established:

$$
A X=J_{m}^{+}
$$

\subsection{FMT reconstruction with CoSaMP}

By exploiting the sparsity characteristic of fluorescent signals, one can formulate the inverse problem of FMT into a $\ell_{1}$ minimization problem and solve it with linear programing algorithms. Unlike this conventional method, the sparse target reconstruction in this paper is accomplished by using iterative greedy algorithm. As an alternative approach to sparse recovery, greedy algorithms find approximate solutions of

$$
\min \|X\|_{0} \quad \text { subject to } \quad\left\|A X-J_{m}^{+}\right\|_{2}^{2} \leq \varepsilon^{2},
$$

where $\|X\|_{0}=\left|\left\{j: X_{j} \neq 0\right\}\right| \ll N$.

Inspired by the restricted isometry property, CoSaMP computes the support of the signal $X$ iteratively and reconstruct the signal using the pseudoinverse. ${ }^{23}$ To identify the locations of the largest components in the target $k$-sparse signal, CoSaMP uses $y=A^{*} J_{m}^{+}$to serve as a proxy for the signal because the energy in each set of $k$ components of the proxy approximates the energy in the corresponding s components of $X$. Consequently, the largest $k$ entries of the proxy point toward the largest $k$ entries of the signal $X$. The algorithm steps for CoSaMP appears as Algorithm 1.

Although it is computationally difficult to check the restricted isometry property of system matrix in FMT, it is possible to recover the sparse signal stably via CoSaMP. To implement reconstruction with greedy algorithms, we need to normalize the columns of system matrix $A$ using an operation $\tilde{\mathrm{A}}=A W$, where $W$ is a diagonal matrix with $1 /\left\|a_{i}\right\|_{2}$ on the main diagonal. With the input $\tilde{\mathrm{A}}$, greedy algorithms produce an approximate 


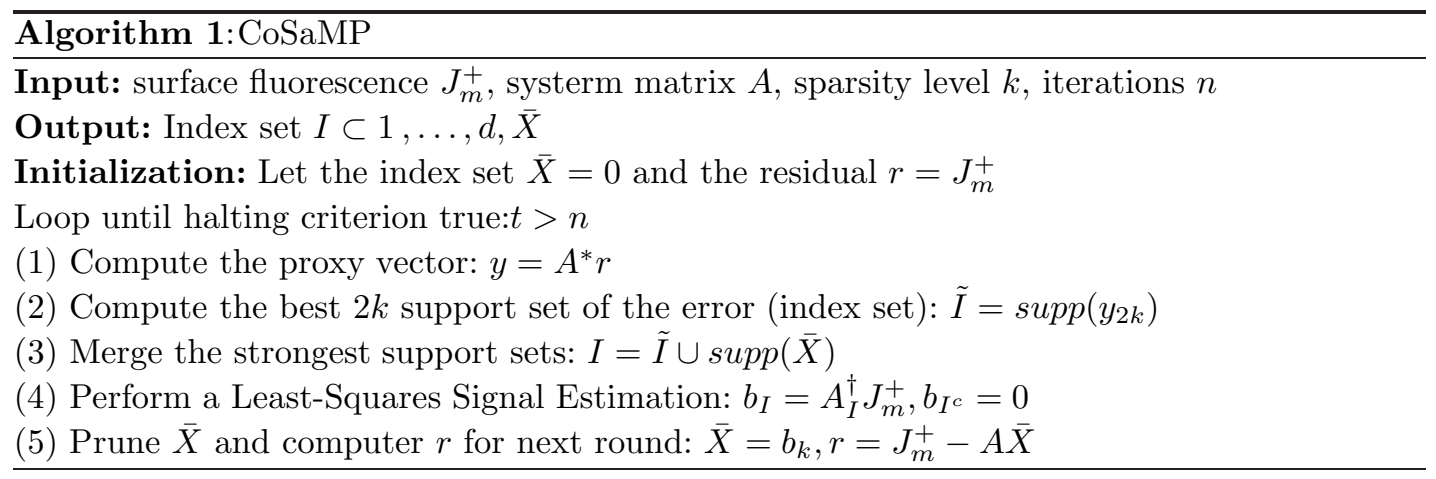

solution $\bar{X}$. Accordingly, we compute the solution of Eq. (7) with $X=W \bar{X}$.

As shown in Algorithm 1, the CoSaMP algorithm requires the sparsity level $k$ as part of its input. Generally, sparse level $k$ is empirically determined by running CoSaMP several times with a range of sparsity levels. In FEM based FMT, $N$ denotes the total number of mesh nodes in the entire region, and $k$ is the number of nodes involved in the target. Consequently, the value of $k$ roughly relates to the target size. By comparing the approximation error incurred by different sparsity levels, we chose the sparsity level $k$ to 2,4 or 8 in the following simulations and experiments.

\section{Experiments and Results}

To validate our algorithm, we conducted simulations on a $3 \mathrm{D}$ digital mouse model ${ }^{29}$ and experiment in a cubic phantom. To evaluate the performance of the iterative CoSaMP algorithm, we compared it to several typical greedy algorithms, i.e., OMP, StOMP, and ROMP. In numerical simulations, single-target and double-target scenarios were considered, respectively. Moreover, the robustness and stability of the proposed greedy method were investigated with simulations.

\subsection{Comparison experiments}

\subsubsection{Experiments setup}

The digital mouse was simulated based on the Digimouse. ${ }^{29}$ The torso part with a height of $35 \mathrm{~mm}$ was chosen as the region to be investigated, including heart, lungs, liver, stomach, kidneys, and muscle, as shown in Fig. 1(a). The optical parameters for different organs are listed in Table $1 .{ }^{15}$ Figure $1(\mathrm{~b})$ illustrates the distribution of 18 excitation sources used in our simulations, which located at the plane of $z=16.5 \mathrm{~mm}$ with $20^{\circ}$ interval. The boundary fluence rate was calculated by solving the $S P_{3}$ model with FEM. For every excitation source, we collected the surface data on the opposite side with a $120^{\circ}$ field of view (FOV). Hence, a total of 18 datasets were assembled for the subsequent reconstruction.

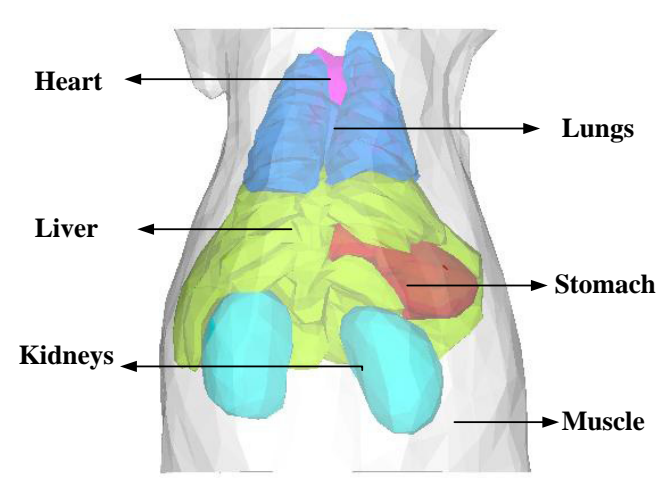

(a)

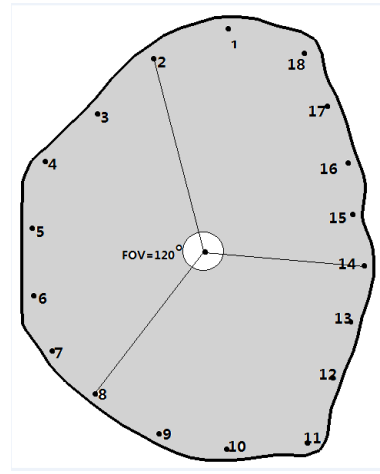

(b)

Fig. 1. (a) Torso of the mouse atlas model (b) The plane of excitation sources at $z=16.5$ mm. For each excitation source, fluorescence is detected at the opposite side with a $120^{\circ} \mathrm{FOV}$. 
Table 1. Optical parameters of the mouse organs.

\begin{tabular}{lcccc}
\hline Tissue & $\mu_{a x}\left(\mathrm{~mm}^{-1}\right)$ & $\mu_{s x}^{\prime}\left(\mathrm{mm}^{-1}\right)$ & $\mu_{a m}\left(\mathrm{~mm}^{-1}\right)$ & $\mu_{s m}^{\prime}\left(\mathrm{mm}^{-1}\right)$ \\
\hline Muscle & 0.0052 & 1.08 & 0.0068 & 1.03 \\
Heart & 0.0083 & 1.01 & 0.0104 & 0.99 \\
Lungs & 0.0133 & 1.97 & 0.0203 & 1.95 \\
Liver & 0.0329 & 0.70 & 0.0176 & 0.65 \\
Kidneys & 0.0660 & 2.25 & 0.0380 & 2.02 \\
Stomach & 0.0114 & 1.74 & 0.0070 & 1.36 \\
\hline
\end{tabular}

\subsubsection{Reconstruction of a single target}

In this section, a cylindrical fluorescent target with $0.8 \mathrm{~mm}$ radius and $1.6 \mathrm{~mm}$ height was placed in the liver with center at $(11.9 \mathrm{~mm}, 6.4 \mathrm{~mm}, 16.4 \mathrm{~mm})$. The fluorescent yield of the fluorescent target was set to be $0.05 \mathrm{~mm}^{-1}$. For the inverse problem, the digital mouse was discretized into 12376 tetrahedral elements and 2604 nodes.

It is well known that iterative methods obtain an approximation close enough to the optimal solution after an infinite number of iterations. By computing the relative error $\left\|A \cdot X-J_{m}^{+}\right\| /\left\|J_{m}^{+}\right\|$caused by the solution at iteration $n$, we can compare the convergence speed of these greedy algorithms and determine the optimal iteration number. As shown in Fig. 2, CoSaMP converged faster than the other comparative algorithms and yielded a smaller relative error in most cases.

Figure 3 shows the reconstruction results of four greedy algorithms in single target case. Table 2 summarized the detailed quantitative results, including the centers of the reconstructed target, location error (LE) and the reconstructed fluorescent yield. As shown in Table 2 and Fig. 3, although all the tested algorithms produce a satisfied result in

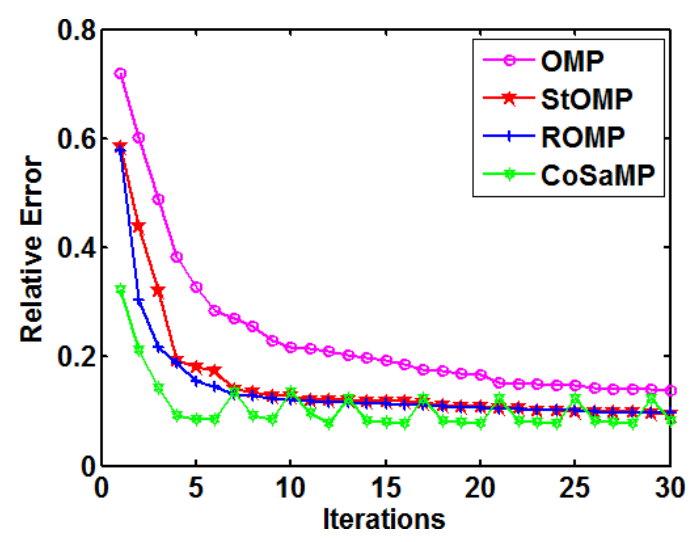

Fig. 2. Comparison of convergence. single target case with LE of $0.4 \mathrm{~mm}$, CoSaMP shows a great improvement in reconstructed fluorescence yield and source distribution.

\subsubsection{Reconstruction of double target}

We also investigated the spatial resolution ability of the proposed greedy algorithm with double-target simulations. Obviously, compared to single-target reconstruction, resolving two close targets is a more challenging task to an inverse algorithm. In the following simulation, two cylindrical targets with uniform size were placed in the liver of the mouse model with a center to center distance of $4.5 \mathrm{~mm}$. The center coordinates of the two targets were $(11.9,10.9,16.4 \mathrm{~mm})$ and $(11.9,6.4,16.4 \mathrm{~mm})$, respectively. Since the radius of the target was $0.8 \mathrm{~mm}$, the actual edge separation between the two targets was $2.9 \mathrm{~mm}$. To better test the performance of the algorithms, we considered two scenarios, i.e., the fluorescence yields ratio between the two targets were $1: 1$ and $2: 1$.

In the first case, the fluorescence yields of the two targets were $0.05 \mathrm{~mm}^{-1}$. Figure 4 and Table 3 show the reconstruction results by the four greedy algorithms. From the reconstructed results, we observed that the results by OMP and ROMP significantly deviate from the actual positions, where LE of one target is up to $3.89 \mathrm{~mm}$ and $4.5 \mathrm{~mm}$, respectively. Both StOMP and CoSaMP can discriminate two targets clearly, but the location accuracy and reconstructed fluorescent yield by CoSaMP are superior to that of StOMP. The LE of Target 2 by CoSaMP is only $0.39 \mathrm{~mm}$, which is smaller than $1.56 \mathrm{~mm}$ by StOMP. Moreover, the relative error in reconstructed fluorescent yield by CoSaMP is within $14 \%$, while that by StOMP is about $38 \%$.

In the second case, the fluorescence yields of the two targets were $0.10 \mathrm{~mm}^{-1}$ and $0.05 \mathrm{~mm}^{-1}$, respectively. Figure 5 illustrate the transverse view of the reconstructions at $z=16.4 \mathrm{~mm}$ and Table 4 presents the quantitative results by the four algorithms. The results of double-targets cases show that OMP and ROMP are incompetent in multiple targets reconstruction. StOMP and CoSaMP generally performed better than the other two algorithms, but CoSaMP outperformed StOMP in the terms of reconstructed fluorescence yields and location accuracy. We observed that the fluorescence yields ratio between the two reconstructed targets 


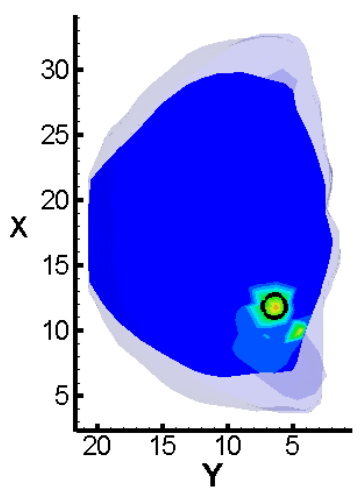

(a)

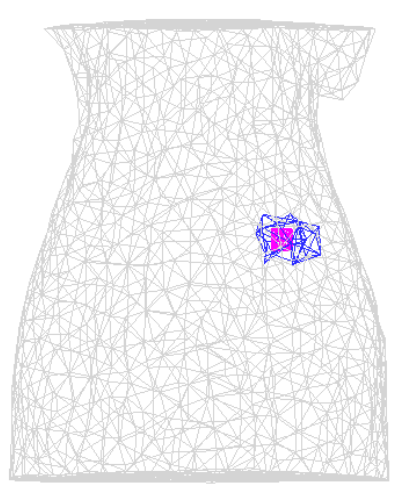

(e)

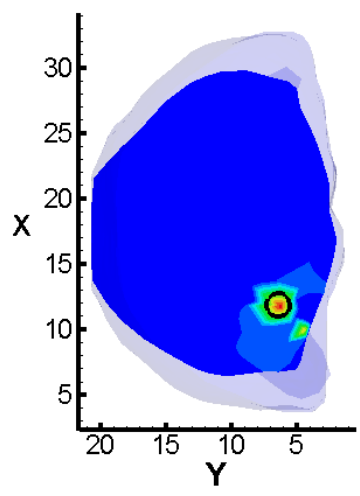

(b)

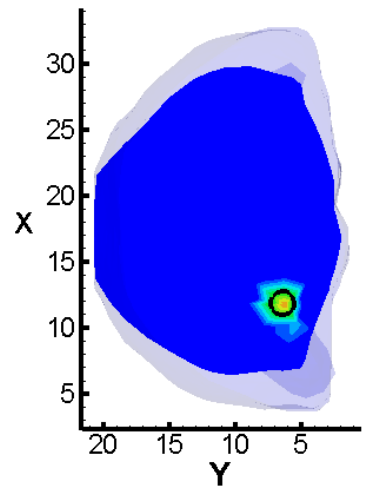

(c)

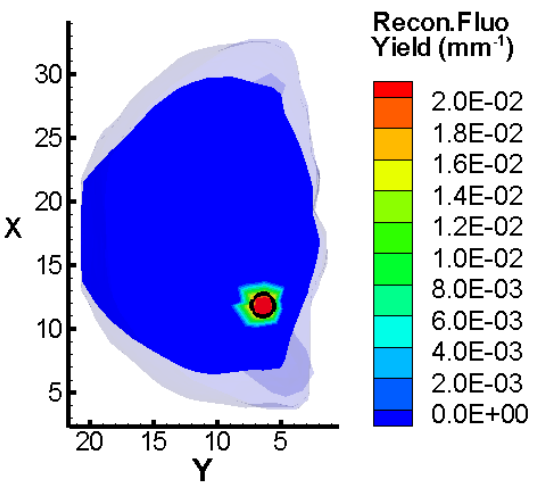

(d)

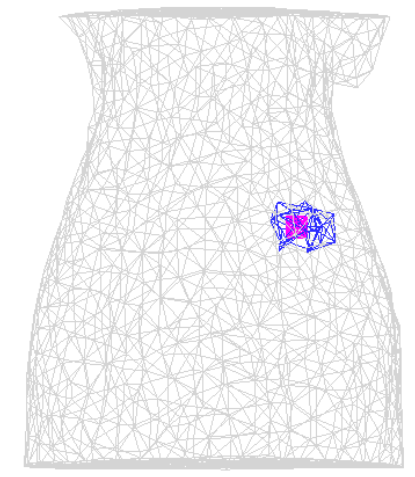

(f)

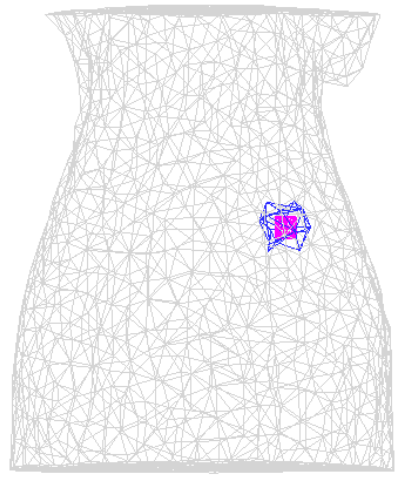

(g)

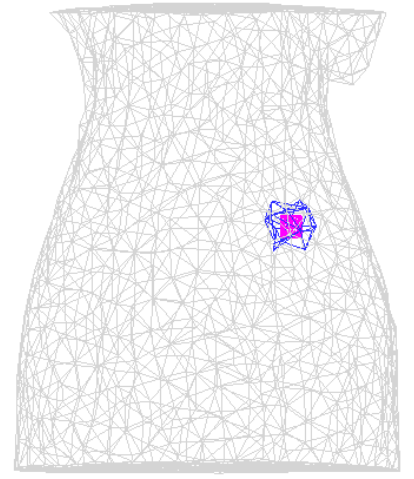

(h)

Fig. 3. Comparison of the reconstruction results in single fluorescent target case. (a), (e) are the results of OMP; (b), (f) are the results of StOMP; (c), (g) are the results of ROMP; (d), (h) are the results of CoSaMP. The figures (a)-(d) are the transverse view of the reconstruction at $z=16.4 \mathrm{~mm}$, the black circle represents the real fluorescent target; (e)-(h) are the $3 \mathrm{D}$ views of the results with node values greater than $70 \%$ of the maximum value.

produced by CoSaMP was 1.9:1. In contrast, the ratio by StOMP was only 1.2:1.

\subsection{Stability analysis}

We evaluated the stability and robustness of the CoSaMP algorithm by taking the influence of noise and the number of excitation sources.

To investigate the influence of measurement noise to the performance of CoSaMP, we separately added $10 \%$ Gaussian noise and $10 \%$ Poisson noise to the simulated measurements in single-target case.
Twenty independent reconstructions were conducted for each kind of noise. The average reconstruction result by CoSaMP is shown in Table 5 . We found that only the reconstructed fluorescent yield was slightly affected by the noise and the location accuracy kept invariable.

The dependence of the reconstruction results on the number of excitation sources was also investigated. The measurable data reduces with the decreasing of the number of excitation sources, and the ill-posedness of FMT reconstruction will accordingly increase. In previous simulations, the

Table 2. Reconstructed results of single fluorescent target.

\begin{tabular}{lccc}
\hline Method & Recon. position center $(\mathrm{mm})$ & LE $(\mathrm{mm})$ & Recon. fluo yield $\left(\mathrm{mm}^{-1}\right)$ \\
\hline OMP & $(11.8,6.3,16.0)$ & 0.40 & 0.016 \\
StOMP & $(11.8,6.3,16.0)$ & 0.40 & 0.023 \\
ROMP & $(11.8,6.3,16.0)$ & 0.40 & 0.016 \\
CoSaMP & $(\mathbf{1 1 . 8 , 6 . 3 , 1 6 . 0 )}$ & $\mathbf{0 . 4 0}$ & $\mathbf{0 . 0 3 8}$ \\
\hline
\end{tabular}




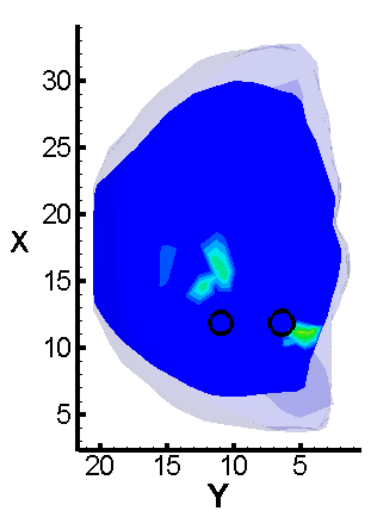

(a)

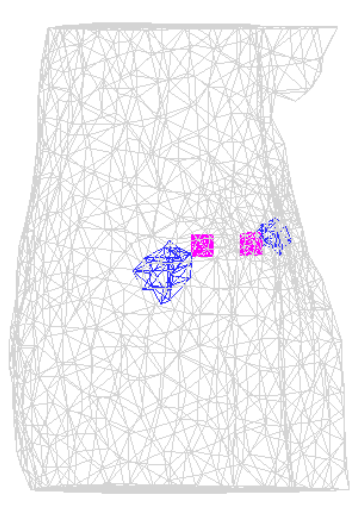

(e)

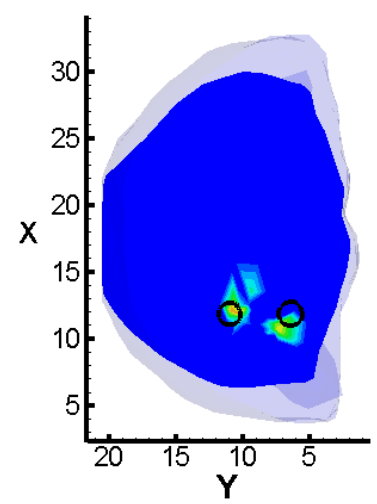

(b)

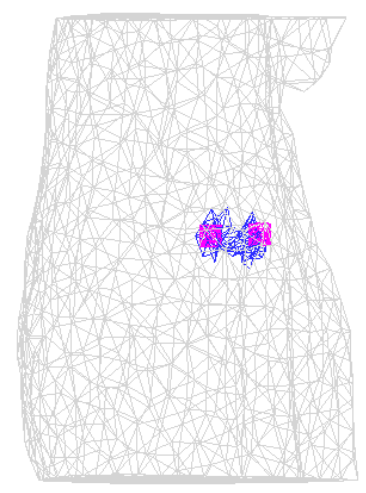

(f)

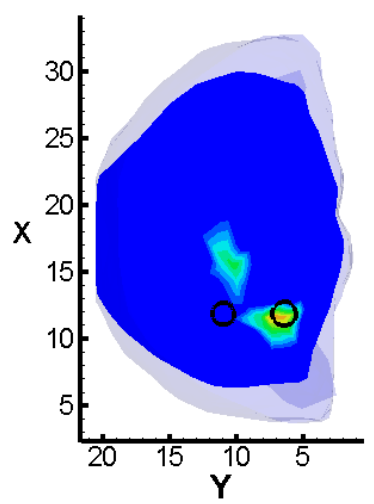

(c)

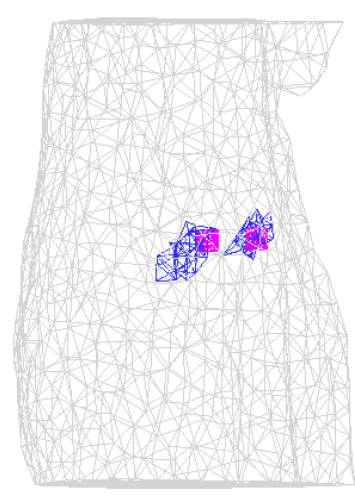

(g)

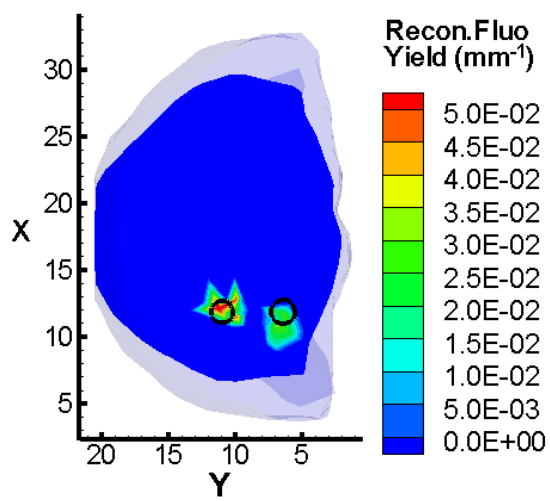

(d)

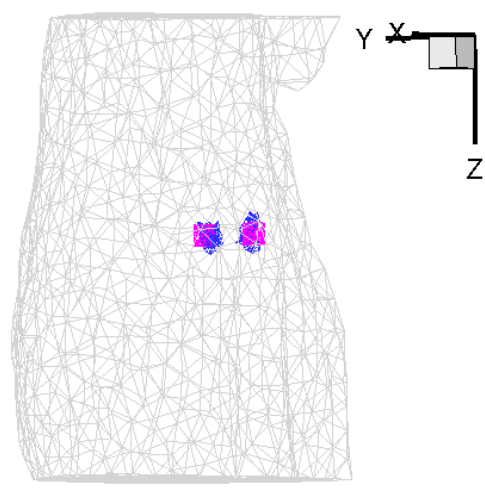

(h)

Fig. 4. Comparison of the reconstruction results in double fluorescent targets case. (a), (e) are the results of OMP; (b), (f) are based on StOMP; (c), (g) are the results of ROMP and (d), (h) are based on CoSaMP. The figures (a)-(d) are the transverse view of the reconstruction at $z=16.4 \mathrm{~mm}$, the black circle represents the real fluorescent target; (e) $-(\mathrm{h})$ are the $3 \mathrm{D}$ views of the results with node values greater than $70 \%$ of the maximum value.

measurement is acquired every $20^{\circ}$ and a total of 18 data sets were assembled for the reconstruction. In this subsection, we decrease the number of excitation sources from 18 to $12,6,3$ and the measurement is obtained every $30^{\circ}, 60^{\circ}, 120^{\circ}$. The detailed reconstruction results with reduced measurements are presented in Table 6. The results reconstructed from measurements with 12 , and 6 excitation nodes are satisfied. However, when the excitation nodes reduce to 3 , the reconstructed target deviated from the actual center. In addition, the reconstructed fluorescent yield gradually declined with the decrease of excitation sources.

Table 3. Reconstructed results of double targets with same fluorescence yields.

\begin{tabular}{lcccc}
\hline Method & Fluo yield $\left(\mathrm{mm}^{-1}\right)$ & Recon. position center $(\mathrm{mm})$ & LE $(\mathrm{mm})$ & Recon. fluo yield $\left(\mathrm{mm}^{-1}\right)$ \\
\hline OMP & 0.05 & $(12.5,9.7,17.2)$ & 1.54 & 0.016 \\
& 0.05 & $(10.4,3.9,19.8)$ & 4.50 & 0.025 \\
\multirow{2}{*}{ StOMP } & 0.05 & $(12.6,10.7,16.8)$ & 0.79 & 0.031 \\
& 0.05 & $(10.7,7.4,16.4)$ & 1.56 & 0.034 \\
ROMP & 0.05 & $(15.4,11.7,17.7)$ & 3.89 & 0.031 \\
& 0.05 & $(11.3,6.7,16.3)$ & 0.62 & 0.033 \\
CoSaMP & 0.05 & $(\mathbf{1 2 . 5 , 1 0 . 7 , 1 6 . 8 )}$ & $\mathbf{0 . 7 9}$ & $\mathbf{0 . 0 4 8}$ \\
& 0.05 & $(\mathbf{1 1 . 7 , 6 . 4 , 1 6 . 7 )}$ & $\mathbf{0 . 3 5}$ & $\mathbf{0 . 0 4 3}$ \\
\hline
\end{tabular}


X. He et al.
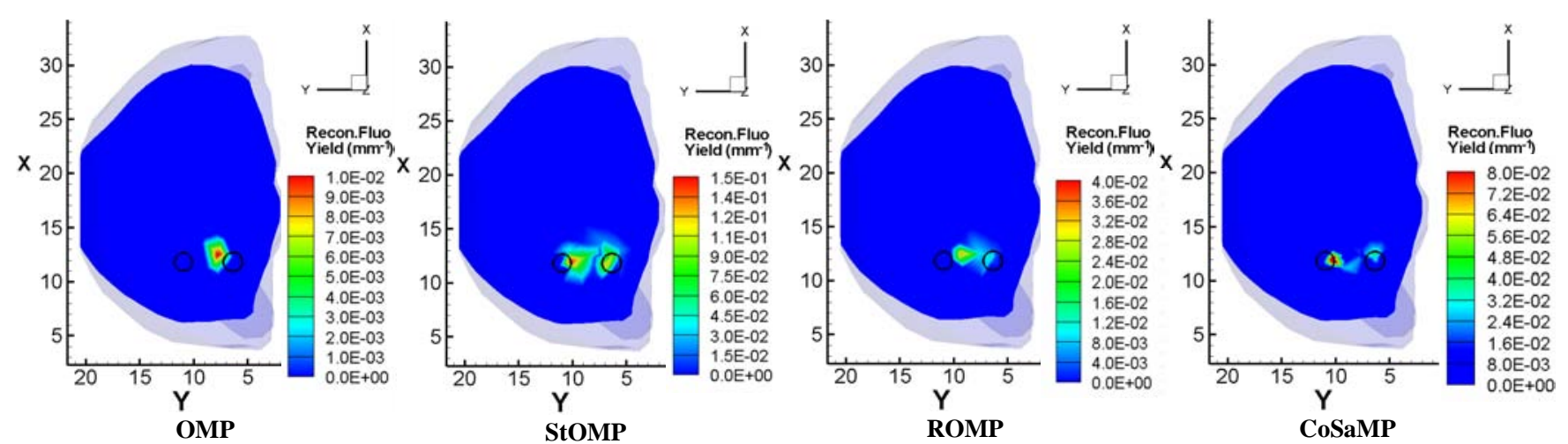

Fig. 5. Reconstruction results in the case of two targets with different fluorescence yields. The true fluorescence yields of the two targets are $0.10 \mathrm{~mm}^{-1}$ and $0.05 \mathrm{~mm}^{-1}$, respectively.

Table 4. Reconstructed results of double targets with different fluorescence yields.

\begin{tabular}{lcccc}
\hline Method & Fluo yield $\left(\mathrm{mm}^{-1}\right)$ & Recon. position center $(\mathrm{mm})$ & LE $(\mathrm{mm})$ & Recon. fluo yield $\left(\mathrm{mm}^{-1}\right)$ \\
\hline OMP & 0.10 & $(9.9,14.8,18.6)$ & 5.01 & 0.003 \\
& 0.05 & $(12.5,7.7,16.2)$ & 1.55 & 0.014 \\
StOMP & 0.10 & $(11.8,9.7,16.8)$ & 1.23 & 0.156 \\
& 0.05 & $(11.7,6.8,16.8)$ & 0.75 & 0.126 \\
ROMP & 0.10 & $(12.4,8.9,16.9)$ & 2.02 & 0.048 \\
& 0.05 & $(12.0,6.7,15.6)$ & 0.81 & 0.028 \\
CoSaMP & 0.10 & $\mathbf{( 1 2 . 0 , 1 0 . 3 , 1 6 . 8 )}$ & $\mathbf{0 . 7 8}$ & $\mathbf{0 . 0 8 9}$ \\
& 0.05 & $(\mathbf{1 2 . 3 , 6 . 4 , 1 6 . 7 )}$ & $\mathbf{0 . 7 7}$ & $\mathbf{0 . 0 4 6}$ \\
\hline
\end{tabular}

Table 5. Reconstruction results by CoSaMP with noisy measurements.

\begin{tabular}{lccc}
\hline Noise type & Recon. position center $(\mathrm{mm})$ & LE $(\mathrm{mm})$ & Recon. fluo yield $\left(\mathrm{mm}^{-1}\right)$ \\
\hline Gaussian & $(11.8,6.3,16.0)$ & 0.40 & 0.028 \\
Poisson & $(11.8,6.3,16.0)$ & 0.40 & 0.026 \\
\hline
\end{tabular}

Table 6. Reconstruction results by CoSaMP with different numbers of excitation sources.

\begin{tabular}{crcc}
\hline Excitation sources & Recon. position center $(\mathrm{mm})$ & LE $(\mathrm{mm})$ & Recon. fluo yield $\left(\mathrm{mm}^{-1}\right)$ \\
\hline 18 & $(11.8,6.3,16.0)$ & 0.40 & 0.038 \\
12 & $(12.4,6.6,16.5)$ & 0.57 & 0.013 \\
6 & $(12.6,6.6,16.4)$ & 0.68 & 0.011 \\
3 & $(13.0,6.8,16.6)$ & 1.21 & 0.009 \\
\hline
\end{tabular}

\subsection{Physical experiments}

The performance of CoSaMP algorithm combined with $S P_{3}$ model was further evaluated with phantom experiment data. In this experiment a polyoxymethylene cubic phantom with a side length of
$20 \mathrm{~mm}$ was utilized and the experimental data was acquired by a prototype FMT imaging system. ${ }^{15}$ The $4000 \mathrm{nM}$ Cy5.5 solution with peak excitation wavelength of $671 \mathrm{~nm}$ was injected into a small hole (radius $1 \mathrm{~mm}$ and height $2 \mathrm{~mm}$ ) drilled in the 


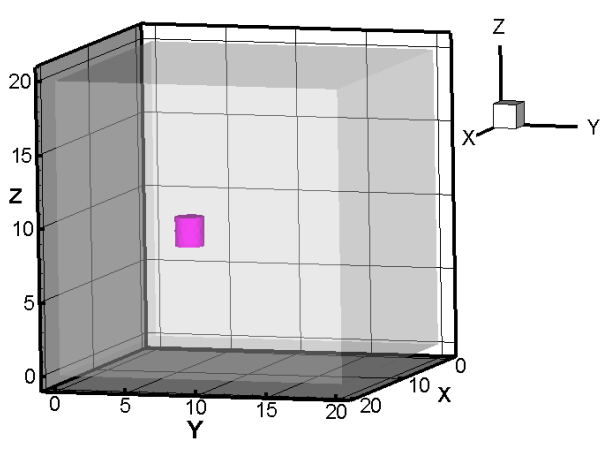

(a)

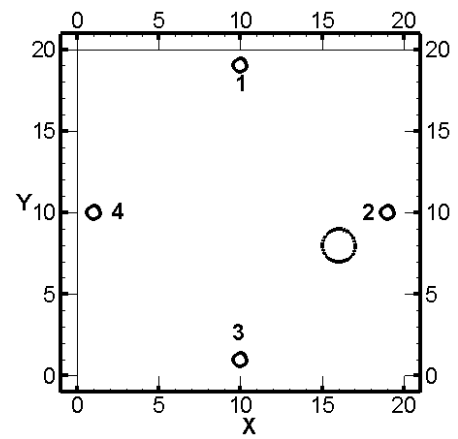

(b)
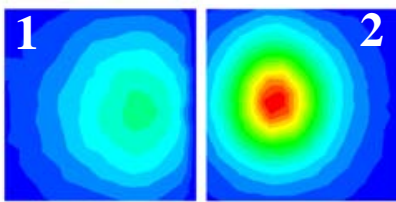

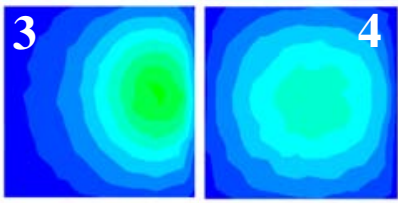

(c)

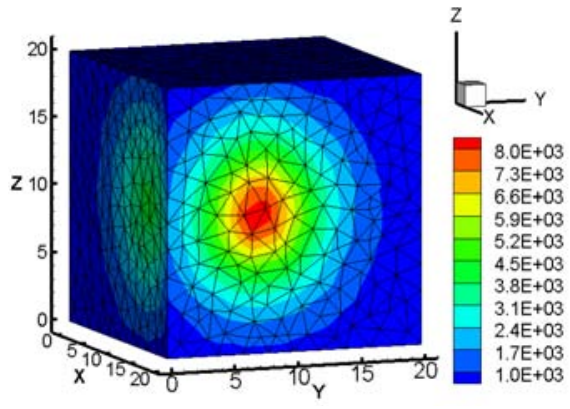

(d)

Fig. 6. (a) The 3D view of the single fluorescent target in the cubic phantom. (b) The $x-y$ view on the $z=10 \mathrm{~mm}$ plane, where the white dots represent the excitation point source positions. (c) The surface data acquired by CCD at four different views. (d) Inverse mesh and photon distribution on the surface.

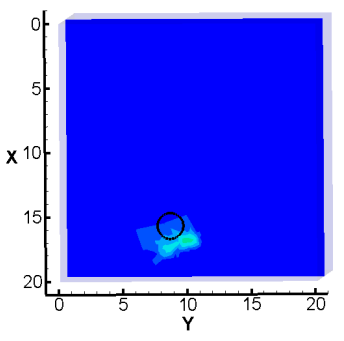

(a)

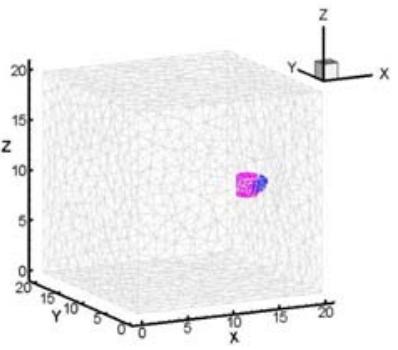

(e)

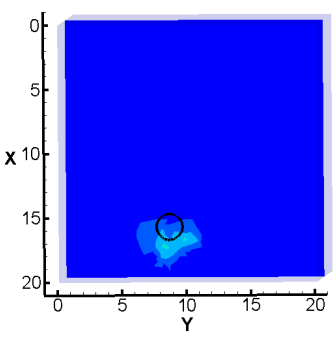

(b)

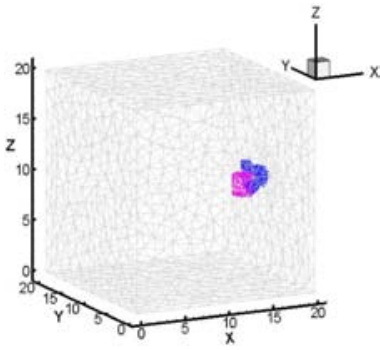

(f)

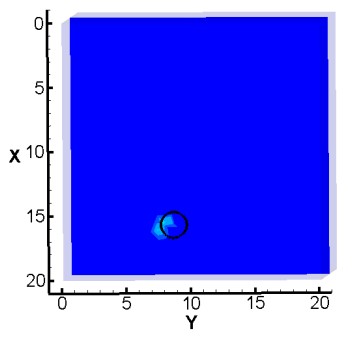

(c)

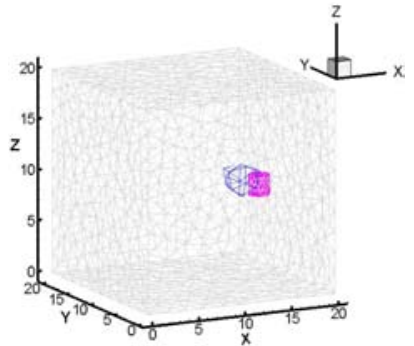

$(\mathrm{g})$

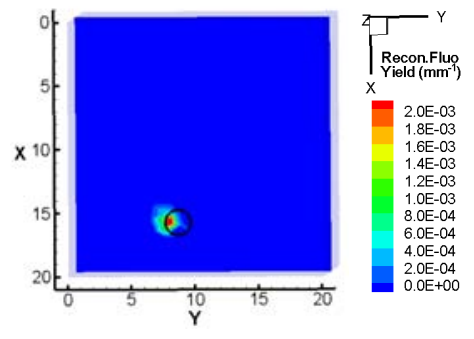

(d)

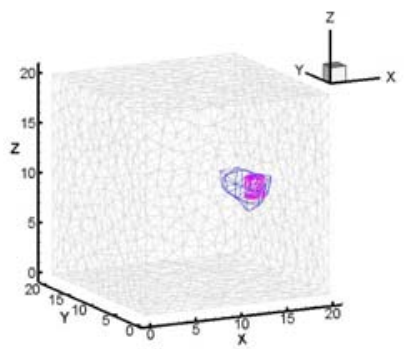

(h)

Fig. 7. Reconstructed results of phantom data. (a)-(d) are the transverse views of the reconstruction by OMP, StOMP, ROMP and CoSaMP on the plane of $z=9.5 \mathrm{~mm}$. The black circle in transverse view represents the true target. (e)-(h) are the 3D isosurface views of the results with top $80 \%$ of the maximum value, where the red cylinder represents the true position of Cy5.5 solution and the blue zone is the reconstructed target. 
phantom to serve as the fluorescent target. The cubic phantom and the source setting is shown in Figs. 6(a) and 6(b), where the center coordinates of the target was $(16,8,9.5 \mathrm{~mm})$. The optical parameters used for excitation wavelength $(671 \mathrm{~nm})$ and emission wavelength $(710 \mathrm{~nm})$ were the same ones in Ref. 15. The fluorescent target was excited by point sources from four different positions at the plane of $z=10 \mathrm{~mm}$. The surface data were acquired with CCD camera from four views by rotating the phantom with an angular increment of $90^{\circ}$, as shown in Fig. 6(c). Figure 6(d) shows the corresponding photon distribution on surface with $2 \mathrm{D}$ to 3D mapping.

For reconstruction, the cubic phantom was discretized into a 3D tetrahedral mesh with 14,780 elements and 2989 nodes. Figure 7 shows the 3D isosurface views of the reconstruction results with top $30 \%$ of the maximum value. The reconstructed Cy 5.5 solution by CoSaMP centered at (16.0, 7.4, $10.2 \mathrm{~mm}$ ), with a LE of $0.92 \mathrm{~mm}$ and fluorescence yield of $0.003 \mathrm{~mm}^{-1}$ which is better than OMP with $1.42 \mathrm{~mm}$ and $0.0011 \mathrm{~mm}^{-1}$, StOMP with $1.44 \mathrm{~mm}$ and $0.0014 \mathrm{~mm}^{-1}$, ROMP with $1.12 \mathrm{~mm}$ and $0.0008 \mathrm{~mm}^{-1}$.

\section{Discussion and Conclusion}

In this paper, FMT reconstruction was regarded as a sparse recovery problem and solved it efficiently by an iterative greedy algorithm. Moreover, $S_{3}$ approximation to RTE was combined to improve the modeling accuracy.

Two groups of comparison simulations were designed to evaluate the reconstruction methods in terms of localization accuracy and fluorescent yield. From the compared results, we found that CoSaMP and StOMP generally performed better than OMP and ROMP in all of the testing cases. The results of double-target cases clearly demonstrated that OMP and ROMP were incompetent in multiple targets reconstruction. Moreover, CoSaMP outperformed StOMP in terms of reconstructed fluorescence yields, location accuracy and multiple target resolving.

To further evaluate the stability and robustness of CoSaMP, the influence of measurement noise and the number of excitation source were taken into consideration. Simulation results showed that measurement noise hardly affected location accuracy of CoSaMP. Only the reconstructed fluorescent yield went down with the increase of noise. In another hand, although measurement decrease caused by reducing excitation nodes also affected the reconstructed fluorescent yield, the reconstruction results were generally acceptable. For example, the location accuracy slightly declined when the excitation nodes reduced from 18 to 6 .

The focus of this paper is to investigate the reconstruction ability of CoSaMP for FMT. For this reason, some widely used strategies for improving reconstruction are not incorporated in our implementation, which include permissible region and multilevel FEM strategy, etc. The simulation and phantom experimental results demonstrate that CosaMP can efficiently reconstruct the sparse target from incomplete and inaccurate measurements even without these strategies. Our future work will combine the advantages of greedy reconstruction algorithm and these auxiliary strategies to further improve the quantitative results.

\section{Acknowledgments}

This work is supported by the National Natural Science Foundation of China (Nos. 61372046, 11571012 and 61401264), the Research Fund for the Doctoral Program of Higher Education of China (New Teachers) (No. 20116101120018), the Science and Technology Plan Program in Shaanxi Province of China (Nos. 2012 KJXX-29 and 2015 KW-002) and the Natural Science Basic Research Plan in Shaanxi Province of China (No. 2015JM6322).

\section{References}

1. A. Ale, V. Ermolayev, E. Herzog, C. Cohrs, M. H. de Angelis, V. Ntziachristos, "Fmt-xct: in vivo animal studies with hybrid fluorescence molecular tomography-x-ray computed tomography," Nat. Methods 9(6), 615-620 (2012).

2. J. K. Willmann, N. Van Bruggen, L. M. Dinkelborg, S. S. Gambhir, "Molecular imaging in drug development," Nat. Rev. Drug Discov. 7(7), 591-607 (2008).

3. C. Darne, Y. Lu, E M. Sevick-Muraca, "Small animal fluorescence and bioluminescence tomography: A review of approaches, algorithms and technology update," Phys. Med. Biol. 59(1), R1-R64 (2014).

4. D. Wang, J. He, H. Qiao, X. Song, Y. Fan, D. Li, "High-performance fluorescence molecular tomography through shape-based reconstruction using 
spherical harmonics parameterization," PloS one 9(4), e94317 (2014).

5. N. Ducros, A. Bassi, G. Valentini, G. Canti, S. Arridge, C. D'Andrea, "Fluorescence molecular tomography of an animal model using structured light rotating view acquisition," J. Biomed. Opt. 18(2), 020503 (2013).

6. J. Ye, Y. Du, Y. An, C. Chi, J. Tian, "Reconstruction of fluorescence molecular tomography via a nonmonotone spectral projected gradient pursuit method," J. Biomed. Opt. 19(12), 126013 (2014).

7. V. Ntziachristos, J. Ripoll, L V. Wang, R. Weissleder, "Looking and listening to light: The evolution of whole-body photonic imaging," Nat. Biotech. 23(3), 313-320 (2005).

8. A. D. Klose, E. W. Larsen, "Light transport in biological tissue based on the simplified spherical harmonics equations," J. Comput. Phys. 220(1), 441-470 (2006).

9. H. Guo, Y. Hou, X. He, J. Yu, J. Cheng, X. Pu, "Adaptive hp finite element method for fluorescence molecular tomography with simplified spherical harmonics approximation," J. Innov. Opt. Health Sci. 7(02) 1350057 (2014).

10. D. Wang, X. Song, J. Bai, "Adaptive-mesh-based algorithm for fluorescence molecular tomography using an analytical solution (Vol. 15, pg. 9722, 2007)," Opt. Express 15(15), 9722-9730 (2007).

11. J. C. Baritaux, K. Hassler, M. Unser, "An efficient numerical method for general regularization in fluorescence molecular tomography," IEEE Trans. Med. Imag. 29(4), 1075-1087 (2010).

12. Y. An, J. Liu, G. Zhang, J. Ye, Y. Du, Y. Mao, C. Chi, J. Tian. "A novel region reconstruction method for fluorescence molecular tomography," IEEE Trans. Biomed. Eng. 62(7), 1818-1826 (2015).

13. D. Han, J. Tian, S. Zhu, J. Feng, C. Qin, B. Zhang, $\mathrm{X}$. Yang, "A fast reconstruction algorithm for fluorescence molecular tomography with sparsity regularization," Opt. Express 18(8), 8630-8646 (2010).

14. J. Shi, F. Liu, H. Pu, S. Zuo, J. Luo, J. Bai, "An adaptive support driven reweighted 11-regularization algorithm for fluorescence molecular tomography," Biomed. Opt. Express 5(11), 40394052 (2014).

15. H. Yi, D. Chen, X. Qu, K. Peng, X. Chen, Y. Zhou, J. Tian, J. Liang, "Multilevel, hybrid regularization method for reconstruction of fluorescent molecular tomography," Appl. Opt. 51(7), 975-986 (2012).

16. W. Xie, Y. Deng, K. Wang, X. Yang, Q. Luo, "Reweighted 11 regularization for restraining artifacts in FMT reconstruction images with limited measurements," Opt. Lett. 39(14), 4148-4151 (2014).
17. D. Zhu, C. Li, "Nonconvex regularizations in fluorescence molecular tomography for sparsity enhancement," Phys. Med. Biol.59(12), 2901 (2014).

18. H. Guo, J. Yu, X. He, Y. Hou, F. Dong, S. Zhang, "Improved sparse reconstruction for fluorescence molecular tomography with $11 / 2$ regularization," Biomed. Opt. Express 6, 1648-1664 (2015).

19. E. J. Candes, T. Tao, "Decoding by linear programming," IEEE Trans. Inform. Theory 51(12), 4203-4215 (2005).

20. J. A. Tropp, A. C. Gilbert, "Signal recovery from random measurements via orthogonal matching pursuit," IEEE Trans. Inform. Theory 53(12), 4655-4666 (2007).

21. D. Han, X. Yang, K. Liu, C. Qin, B. Zhang, X. Ma, J. Tian, "Efficient reconstruction method for 11 regularization in fluorescence molecular tomography," Appl. Opt. 49(36), 6930-6937 (2010).

22. J. Ye, C. Chi, Z. Xue, P. Wu, Y. An, H. Xu, S. Zhang, J. Tian, " Fast and robust reconstruction for fluorescence molecular tomography via a sparsity adaptive subspace pursuit method," Biomed. Opt. Express 5(2), 387-406 (2014).

23. D. Needell, J. Tropp, "CoSaMP: Iterative signal recovery from incomplete and inaccurate samples," Appl. Comput. Harmon. Anal. 26(3), 301-321 (2008).

24. M. A. Davenport, D. Needell, M. B. Wakin, "Signal space cosamp for sparse recovery with redundant dictionaries," IEEE Trans. Inform. Theory 59(10), 6820-6829 (2013).

25. Y. Yongdou, Y. Jianqiao, W. Yuyong, L. Xia, C. Tingting, "A improved cosamp algorithm based on correlation coefficient for compressed sensing image reconstruction," J. Comput. Inform. Syst. 9(18), 7325-7331 (2013).

26. A. D. Klose, B. J. Beattie, H. Dehghani, L. Vider, C. Le, V. Ponomarev, R. Blasberg, "In vivo bioluminescence tomography with a blocking-off finitedifference sp3 method and mri/ct coregistration," Med. Phys. 37(1), 329-338 (2010).

27. M. Chu, H. Dehghani, "Image reconstruction in diffuse optical tomography based on simplified spherical harmonics approximation," Opt. Express 17(26), 24208-24223 (2009).

28. Y. Lu, A. Douraghy, H. B. Machado, D. Stout, J. Tian, H. Herschman, A. F. Chatziioannou, "Spectrally resolved bioluminescence tomography with the third-order simplified spherical harmonics approximation," Phys. Med. Biol. 54(21), 6477 (2009).

29. B. Dogdas, D. Stout, A. F. Chatziioannou, R. M. Leahy, "Digimouse: A 3d whole body mouse atlas from ct and cryosection data," Phys. Med. Biol. $\mathbf{5 2}(3), 577$ (2007). 